\title{
Endotoxin tolerance induced by lipopolysaccharide preconditioning protects against surgery-induced cognitive impairment in aging mice
}

\author{
ZHIJIE ZHANG ${ }^{1-3}$, MUHUO JI ${ }^{2,4}$, YANLIN LIAO ${ }^{2}$, JIANJUN YANG ${ }^{2,4}$ and JUN GAO ${ }^{1}$ \\ ${ }^{1}$ Department of Neurobiology, Key Laboratory of Human Functional Genomics of Jiangsu, Nanjing Medical University, \\ Nanjing, Jiangsu 211166; ${ }^{2}$ Department of Anesthesiology, Jinling Clinical Medical College of \\ Nanjing Medical University, Nanjing, Jiangsu 210003; ${ }^{3}$ Department of Anesthesiology, Huai'an First People's Hospital,

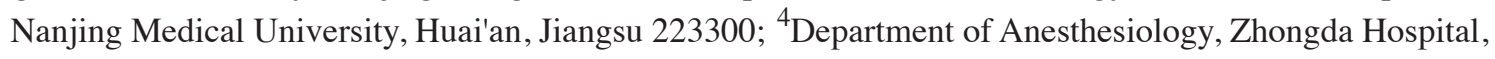 \\ Medical School, Southeast University, Nanjing, Jiangsu 210009, P.R. China
}

Received August 22, 2017; Accepted November 9, 2017

DOI: $10.3892 / \mathrm{mmr} .2018 .8370$

\begin{abstract}
Postoperative cognitive dysfunction (POCD) is a clinical syndrome characterized by varying degrees of cognitive functional decline in patients following major surgery. Inflammation and a dysregulated innate immune system exert broad effects in the periphery and central nervous system, yet the mechanisms underlying POCD remain poorly understood and without effective therapy. It has been reported that modulation of the dysregulated inflammatory response with low-dose lipopolysaccharide (LPS) preconditioning, a phenomenon additionally referred to as endotoxin tolerance, has the potential to reduce neuroinflammation, blood-brain barrier disruption, and cognitive impairment in a number of disease states. Therefore, it was hypothesized that endotoxin tolerance induced by LPS preconditioning may protect against surgery-induced cognitive impairment in aging mice. Using a mouse model of surgery-induced cognitive decline, the present study demonstrated that exploratory laparotomy caused a significant impairment in hippocampal-dependent memory. Notably, one application of low-dose LPS preconditioning at $24 \mathrm{~h}$ prior to surgery improved the cognitive impairment and abolished the signs of neuroinflammation in the hippocampus following surgery. However, LPS injection at $6 \mathrm{~h}$ or immediately prior to surgery did not confer such beneficial effects, suggesting that the effects of LPS-induced endotoxin tolerance may depend on the time of application. In conclusion, the results of the present study suggested that low-dose
\end{abstract}

Correspondence to: Professor Jun Gao, Department of Neurobiology, Key Laboratory of Human Functional Genomics of Jiangsu, Nanjing Medical University, 101 Longmian Road, Jiangning, Nanjing, Jiangsu 211166, P.R. China

E-mail: gaojun19750215@126.com

Key words: surgery, aging, cognitive impairment, neuroinflammation, endotoxin tolerance
LPS preconditioning may markedly alleviate surgery-induced neuroinflammation and cognitive impairment in aging mice, which may provide a novel approach to preventing POCD and, potentially, other forms of memory impairment.

\section{Introduction}

Postoperative cognitive dysfunction (POCD) is a clinical syndrome characterized by varying degrees of cognitive functional decline in patients following major surgery, particularly in the elderly (1-3). POCD affects a considerable proportion of the surgical population worldwide, leading to impaired postoperative rehabilitation, decreased quality of life, and even increased mortality (1). The incidence of POCD varies extensively, from $41-75 \%$ at 7 days to $18-45 \%$ at 3 months postoperatively across different studies (1-4). Considering advances in surgical and anesthetic techniques, and in combination with the aging population, POCD has become an area of focus for anesthesia researchers. Therefore, the prevention and treatment of POCD has become a notable public health issue.

The mechanisms underlying surgery-induced cognitive impairment are multifaceted, although numerous lines of evidence implicate inflammation as a potential driving factor that serves a central role (5-8). Surgery and tissue damage trigger an initial peripheral innate immune response by releasing pro-inflammatory cytokines, including nitric oxide (NO), tumor necrosis factor- $\alpha$ (TNF- $\alpha)$, interleukin- $1 \beta$ (IL-1 $\beta$ ), IL-6, and late cytokine high-mobility group box 1 (HMGB1) (6-9). This systemic inflammatory milieu further leads to transient endothelial dysfunction and disrupts the integrity of the blood-brain barrier (BBB), facilitating the migration of macrophages into the brain parenchyma, and inducing subsequent neuroinflammation with neuronal impairment and ensuing cognitive dysfunction (9). Thus, from a clinical perspective, suppressing inflammation represents a legitimate way to reduce surgery-induced cognitive impairment. In support of this hypothesis, it has been reported that prophylactic administration of either a 
monoclonal antibody to TNF- $\alpha$, or disabling HMGB1 with an inhibitory monoclonal antibody, improves post-surgical cognitive decline (10-12). However, direct neutralization of these pro-inflammatory mediators may lead to undesirable side effects. It is possible that therapeutics directed to the early physiopathological conditions that derive from this initial pro-inflammatory response, while not directly antagonizing them, may be more efficient in preventing the occurrence of POCD.

It has been demonstrated that preconditioning with low-dose lipopolysaccharide (LPS; a bacterial endotoxin) has protective effects against subsequent insults, as evidenced by reduced neuroinflammation, $\mathrm{BBB}$ disruption and cognitive decline, a phenomenon termed endotoxin tolerance (13-15). On the basis of these findings, it was hypothesized that endotoxin tolerance induced by LPS preconditioning may abolish the exacerbated inflammation within the brain, and thus protect against surgery-induced cognitive impairment in aging mice.

\section{Materials and methods}

Animals. A total of 172 C57BL/6 male mice (12-14 months; 24-36 g) were purchased from the Animal Center of Nanjing Medical University (Nanjing, China). The mice were housed in pairs in a colony room maintained at $24 \pm 1^{\circ} \mathrm{C}$ and $40-50 \%$ relative humidity with a 12 -h light-dark cycle (lights on at 07:00 a.m.). Mouse chow and water were available ad libitum. All studies were approved by the Institutional Animal Care and Use Committee of Jinling Clinical Medical College of Nanjing Medical University, and met the Guide for the Care and Use of Laboratory Animals from the National Institutes of Health (NIH; Bethesda, MD, USA) guidelines for the Use of Experimental Animals in research.

Animal grouping and surgical model. Mice were randomly assigned to the following groups: Control $(n=22)$; surgery $(n=30)$; surgery + repeated LPS $(-72 h)(n=30)$; surgery + LPS (-24 h) $(n=30)$; surgery + LPS $(-6$ h) $(n=30)$; and surgery + LPS $(0 \mathrm{~h})$ groups $(\mathrm{n}=30)$. Exploratory laparotomy was performed as previously described $(16,17)$. Mice were allowed to acclimate for 2 weeks prior to the experiments. The surgery was performed under $1.5 \%$ isoflurane anesthesia to mimic exploratory abdominal surgery in humans. A median abdominal incision ( 1-cmlong vertical incision) was made to allow for penetration of the peritoneal cavity. Thereafter, the investigator inserted blunt forceps into the opening and explored the viscera, intestines and musculature. Next, sterile 4-0 chromic gut sutures were used to close the peritoneal lining and skin. The wound was dressed with polysporin (Pfizer, Inc., New York, NY, USA). The animals were maintained under isoflurane anesthesia during the $10 \mathrm{~min}$ of the surgical procedure. Sham controls received neither anesthesia nor surgery. In the present study, all efforts were made to minimize animal suffering and reduce the number of animals used.

LPS administration. All injections were performed intraperitoneally (i.p.) at a volume of $5 \mathrm{ml} / \mathrm{kg}$ body weight. LPS (from Escherichia coli 0111:B4, L-2630; Sigma-Aldrich;
Merck KGaA, Darmstadt, Germany) was dissolved in $0.9 \%$ $\mathrm{NaCl}$. The dose $(0.2 \mathrm{mg} / \mathrm{kg})$ of LPS was based on previous studies, in which it was demonstrated that LPS preconditioning abolished the exacerbated inflammation and protected against cognitive impairment in other animal models $(13,14)$. Animals in the control group received an injection of $0.9 \%$ $\mathrm{NaCl}$ with the same regimen. All mice were injected with LPS or saline between 8.00 and 9.00 a.m. Mice were sacrificed and tissue was collected at 24, 72 and $168 \mathrm{~h}$ following preconditioning. Tissue was additionally collected from six untreated mice to include as a baseline control group. In the present study, no animal succumbed during the observation period in all groups. The flow chart of the experimental protocol is presented in Fig. 1.

Open field test. An open field test was performed to evaluate the exploratory and anxiety behaviors. Mice were placed individually in the center of a clear Plexiglas box $(50 \times 50 \times 40 \mathrm{~cm})$ and left free to explore the environment for $5 \mathrm{~min}$. Total movements and time spent in the center of the open field were recorded during a 5-min exploration time period. The behavior of the mice was recorded using a video camera (Software Technology Co., Ltd., Ningbo, China). The apparatus was cleaned with $70 \%$ ethanol prior to testing each mouse to avoid the presence of olfactory cues.

Fear conditioning tests. Fear conditioning tests were performed to evaluate fear learning, as previously described $(16,17)$. Mice were placed into a conditioning chamber $(32 \times 25 \times 25 \mathrm{~cm})$, with a stainless-steel shock grid floor. Mice learn to associate an environment (context) with a conditional stimulus (CS), for example, a tone, and an unpleasant stimulus [foot shock; unconditional stimulus (US)]. The training paradigm was as follows: Tone at $75 \mathrm{~dB}$ for $30 \mathrm{sec}$; shock at $0.75 \mathrm{~mA}$ for $2 \mathrm{sec}$. Contextual memory was tested $24 \mathrm{~h}$ subsequent to the training. The animals were placed back in the original training chamber to monitor freezing behavior, which was defined as an absence of any movement for $>3 \mathrm{sec}$. The cued fear memory was tested $2 \mathrm{~h}$ subsequently in a novel context with a continuous 3-min training tone presentation to monitor freezing behavior.

ELISA analysis. The concentrations of TNF- $\alpha$ (cat. no. 22351), IL-1 $\beta$ (cat. no. 23107), IL-6 (cat. no. 21724), and IL-10 (cat. no. 26271) in the hippocampus were determined using ELISA kits (North China Institute of Science and Technology, Beijing, China), according to the manufacturer's instructions, as previously described $(16,17)$. Mice were sacrificed with an i.p. injection of $2 \%$ sodium pentobarbital $(60 \mathrm{mg} / \mathrm{kg})$ and the hippocampus was collected, separated, and placed in a homogenizer. Homogenates were centrifuged at $10,000 \mathrm{x} \mathrm{g}$ for $10 \mathrm{~min}$ at $4^{\circ} \mathrm{C}$. The quantity of TNF- $\alpha$, IL-1 $\beta$, IL-6 and IL-10 in each sample was standardized to its protein content.

Immunofluorescence. Under deep isoflurane anesthesia, mice were perfused transcardially with normal saline, followed by $4 \%$ paraformaldehyde (PFA) in PBS for $15 \mathrm{~min}$. Brains were harvested and post-fixed in $4 \%$ PFA overnight at room temperature and then with $30 \%$ sucrose for $24 \mathrm{~h}$ at $4^{\circ} \mathrm{C}$. 
Brains were freeze-mounted in optimal cutting temperature $\left(-20^{\circ} \mathrm{C}\right)$ embedding medium, cut into $10-\mu$ m-thick sections using a cryostat, and mounted on slides. Slices were blocked with $3 \%$ bovine serum albumin (Thermo Fisher Scientific, Inc., Waltham, MA, USA) for $1 \mathrm{~h}$ at room temperature. The sections were incubated with a rat anti-allograft inflammatory factor 1 [IBA1 (a marker of microglial activation), 1:200, cat. no. ab48004; Abcam, Cambridge, UK] antibody overnight at $4^{\circ} \mathrm{C}$, followed by a $1 \mathrm{~h}$ incubation with the secondary antibodies [Cy3-conjugated donkey anti-rat immunoglobulin G (1:300, cat. no. sc53682; Santa Cruz Biotechnology, Inc., Dallas, TX, USA] at room temperature. Following three washes for $5 \mathrm{~min}$ at room temperature using PBS, sections were counterstained with DAPI for $15 \mathrm{~min}$ at room temperature, mounted on glass slides and coverslipped with fluorescence mounting medium. A total of three independent microscopic fields in each section were randomly acquired in the hippocampal CA1, CA3 and dentate gyrus regions, and three sections per mouse were imaged. Images were processed, and the area of the microglia was quantified using ImageJ software (version 1.50i; National Institutes of Health).

Statistical analysis. Statistical analysis was performed using SPSS 16.0 for Windows (SPSS, Inc., Chicago, IL, USA). Data are presented as the mean \pm standard error of the mean. Multiple comparisons were analyzed using one-way analysis of variance followed by the post hoc Tukey test. $\mathrm{P}<0.05$ was considered to indicate a statistically significant difference.

\section{Results}

Single low-dose LPS preconditioning at $24 \mathrm{~h}$ prior to surgery reverses surgery-induced cognitive impairment. To first exclude possible impairments in locomotor activity and exploratory behavior following surgery, mice were evaluated in an open field arena prior to the contextual assessment. As presented in Fig. 2A and B, no alterations were observed in the total distance and time spent in the center of the arena between any group (total distance: $\mathrm{F}_{(5,66)}=0.546, \mathrm{P}=0.740$; time spent in the center: $\left.\left.\mathrm{F}_{(5,66}\right)=1.871, \mathrm{P}=0.111\right)$.

To assess whether surgery induced cognitive impairments, cognitive function was assessed by fear conditioning tests. As revealed in Fig. 2C and D, the contextual fear response was significantly decreased in mice subjected to surgery compared with the control group. Notably, a single LPS preconditioning treatment at $24 \mathrm{~h}$ prior to surgery restored freezing behavior, an indicator of memory retention in rodents when performed at day 7 following surgery $\left(\mathrm{F}_{(5,66)}=5.274, \mathrm{P}<0.001\right.$; Fig. $\left.2 \mathrm{C}\right)$. Repeated low-dose LPS administration did not reverse surgery-induced cognitive impairment when compared with the control group $(\mathrm{P}=0.099)$. However, no significant difference was observed in post-tone freezing time in the auditory-cued fear test between groups $\left(\mathrm{F}_{(5,66)}=1.460, \mathrm{P}=0.215\right.$; Fig. 2D), suggesting that surgery impaired hippocampal-dependent memory (5).

Low-dose LPS preconditioning reverses the signs of neuroinflammation, depending on the regime of LPS administration. In general, repeated injections of LPS at an increasing dose
A

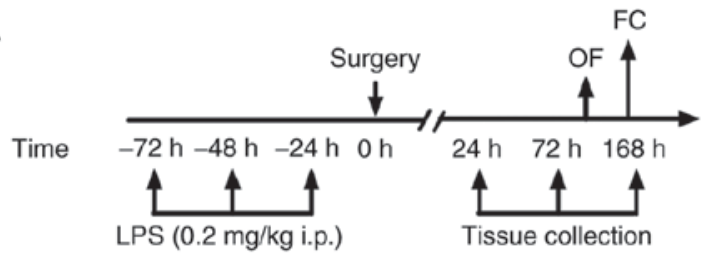

B

Time

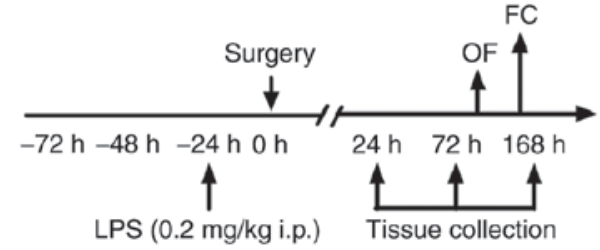

C

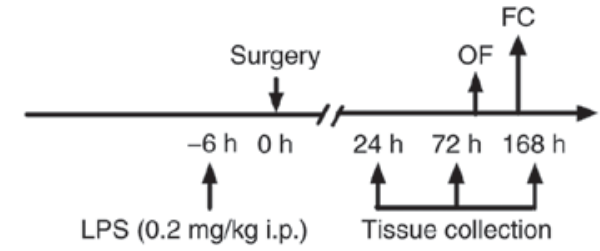

D

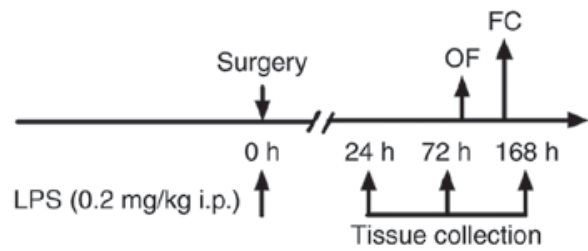

Figure 1. Experimental protocols used in the present study. (A) Schematic representation of the protocol and timeline in the surgery + repeated LPS $(-72 \mathrm{~h}$ ) group; (B) schematic representation of the protocol and timeline in the surgery + LPS (-24 h) group; (C) schematic representation of the protocol and timeline in the surgery + LPS (-6 h) group; and (D) schematic representation of the protocol and timeline in the surgery + LPS $(0 \mathrm{~h})$ group. LPS, lipopolysaccharide; OF, open field; FC, fear conditioning; i.p., intraperitoneal.

may provide greater suppression of pro-inflammatory responses. Therefore, LPS was first administered at $72 \mathrm{~h}$ prior to surgery for 3 consecutive days to investigate whether this regime may decrease the signs of neuroinflammation and consequently improve cognitive impairment following surgery. As presented in Fig. 3, repeated LPS administration significantly decreased the hippocampal expression levels of pro-inflammatory mediators, including: TNF- $\alpha$, IL- $1 \beta$ and IL-6, at 24 h post-surgery $\left(\mathrm{TNF}-\alpha\right.$ : $\mathrm{F}_{(4,25)}=4.730, \mathrm{P}=0.001$; IL-1 $\left.\beta: \mathrm{F}_{(4,25)}=5.435, \mathrm{P}<0.001 ; \mathrm{IL}-6: \mathrm{F}_{(4,25)}=3.534, \mathrm{P}=0.008\right)$. However, repeated LPS administration did not significantly alter the hippocampal levels of the anti-inflammatory mediator IL-10 at $24 \mathrm{~h}$ post-surgery (IL-10: $\left.\mathrm{F}_{(4,25)}=0.785, \mathrm{P}=0.587\right)$. In addition, it was observed that there was no difference in the hippocampal levels of TNF- $\alpha$, IL-1 $\beta$, IL- 6 and IL-10 among the groups at $72 \mathrm{~h}$ post-surgery, returning to the baseline levels as in the control mice. Based on these results, and to minimize the number of animals used, cytokine levels were only measured at 24 and $72 \mathrm{~h}$ post-surgery in the subsequent experiments.

Although repeated LPS administration downregulated the signs of neuroinflammation, it was observed that this regime did not confer beneficial cognitive outcomes. Subsequently, the present study tested the hypothesis that a single low-dose LPS administration at $24 \mathrm{~h}$ prior to surgery may decrease 
A

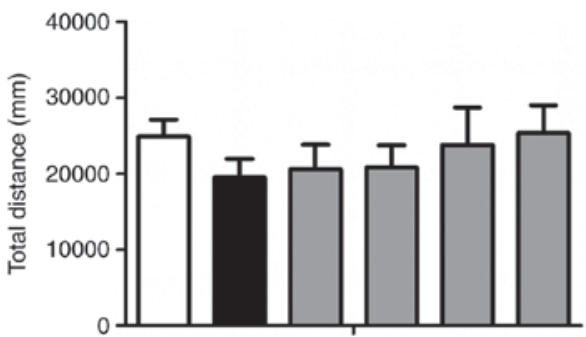

C

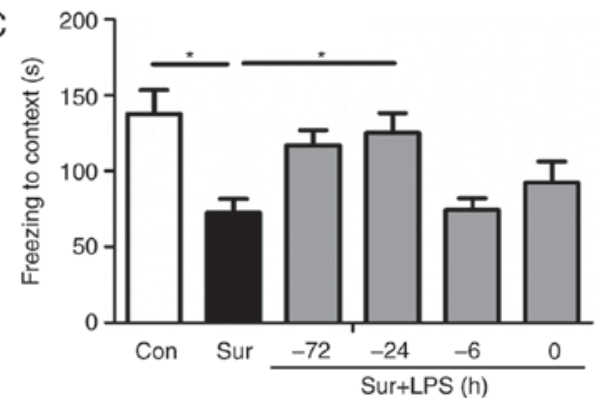

B

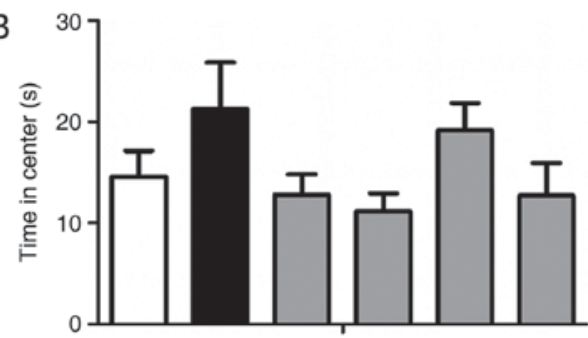

D

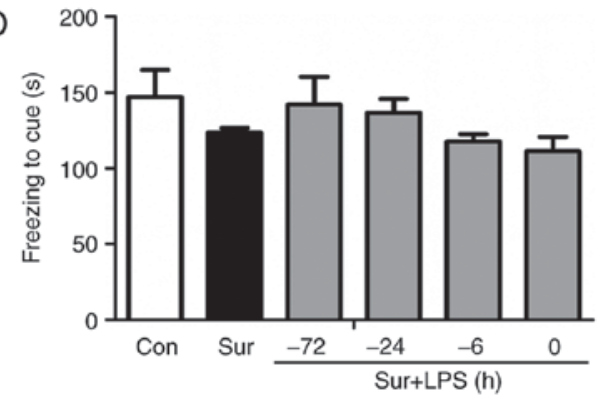

Figure 2. Single low-dose LPS preconditioning at $24 \mathrm{~h}$ prior to surgery reverses surgery-induced cognitive impairment. No alterations were observed in the (A) total distance or (B) time spent in the center of the arena between groups. (C) A single LPS preconditioning treatment at 24 h prior to surgery improved memory impairment. (D) No significant difference was observed in post-tone freezing time in the auditory-cued fear test between groups. ${ }^{*}<0.05$. Con, control; Sur, surgery; LPS, lipopolysaccharide.
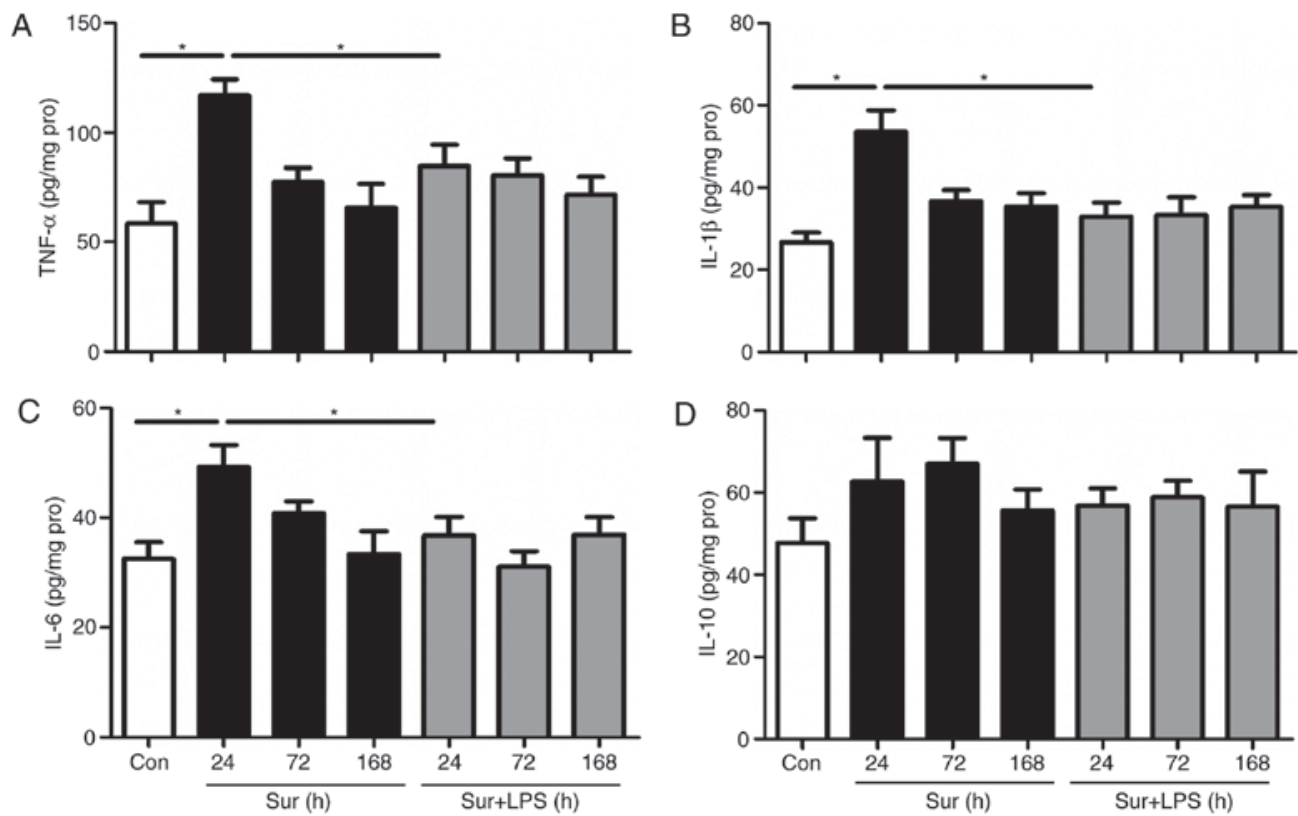

Figure 3. Effects of repeated LPS administration at $72 \mathrm{~h}$ prior to surgery on the hippocampal levels of cytokines. Repeated LPS administration significantly decreased hippocampal levels of (A) TNF- $\alpha$, (B) IL-1 $\beta$ and (C) IL-6 at 24 h post-surgery. Repeated LPS administration did not significantly alter the hippocampal level of (D) IL-10 at 24 h post-surgery. "P<0.05. Con, control; Sur, surgery; LPS, lipopolysaccharide; IL, interleukin; TNF, tumor necrosis factor.

the levels of pro-inflammatory mediators, in addition to reversing cognitive impairment. As expected, one single low-dose treatment with LPS at $24 \mathrm{~h}$ prior to surgery significantly reduced the hippocampal levels of TNF- $\alpha$, IL-1 $\beta$ and IL-6 at $24 \mathrm{~h}$ post-surgery $\left(\mathrm{TNF}-\alpha: \mathrm{F}_{(4,25)}=9.254, \mathrm{P}<0.001\right.$; IL-1 $\beta: \mathrm{F}_{(4,25)}=6.598, \mathrm{P}=0.001$; IL-6: $\mathrm{F}_{(4,25)}=4.410, \mathrm{P}=0.008$; Fig. 4A-C). Similarly, a single low dose of LPS at $24 \mathrm{~h}$ prior to surgery did not significantly alter the hippocampal level of the anti-inflammatory mediator IL-10 following surgery (IL-10: $\mathrm{F}_{(4,25)}=0.890, \mathrm{P}=0.484$; Fig. 4D).
To further investigate whether a shorter interval between LPS administration and surgery may produce similar effects to the $24 \mathrm{~h}$ regime, LPS was administered $6 \mathrm{~h}$ prior to surgery. A single low-dose LPS administration at $6 \mathrm{~h}$ prior to surgery did not alter the cytokine levels (TNF- $\alpha$ : $\mathrm{F}_{(4,25)}=10.713, \mathrm{P}<0.001$; IL-1 $\beta: \mathrm{F}_{(4,5)}=9.9655, \mathrm{P}<0.001$; IL-6: $\mathrm{F}_{(4,25)}=5.058, \mathrm{P}=0.004$; IL-10: $F_{(4,25)}=1.434, P=0.252$; Fig. 5).

To evaluate whether LPS administration can aggravate the inflammatory response to surgical trauma, low-dose LPS was administered immediately prior to surgery. Unexpectedly, 
A

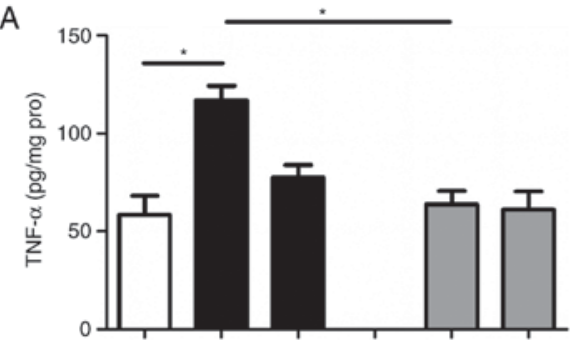

C

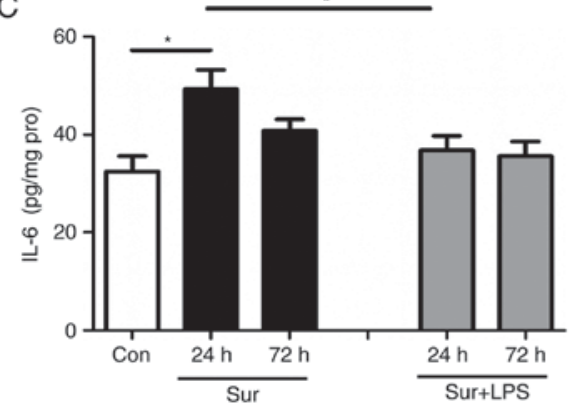

B

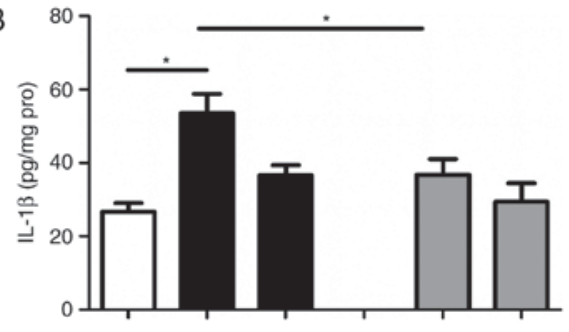

D

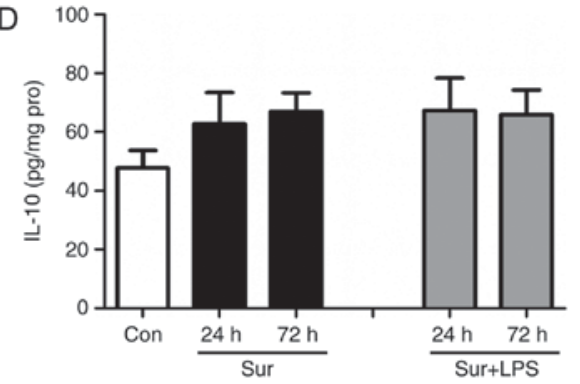

Figure 4. Effects of LPS administration at $24 \mathrm{~h}$ prior to surgery on the hippocampal levels of cytokines. LPS administration significantly decreased hippocampal levels of (A) TNF- $\alpha$, (B) IL-1 $\beta$, and (C) IL-6 at 24 h post-surgery. LPS administration did not significantly alter the hippocampal level of (D) IL-10. "P<0.05. Con, control; Sur, surgery; LPS, lipopolysaccharide; IL, interleukin; TNF, tumor necrosis factor.
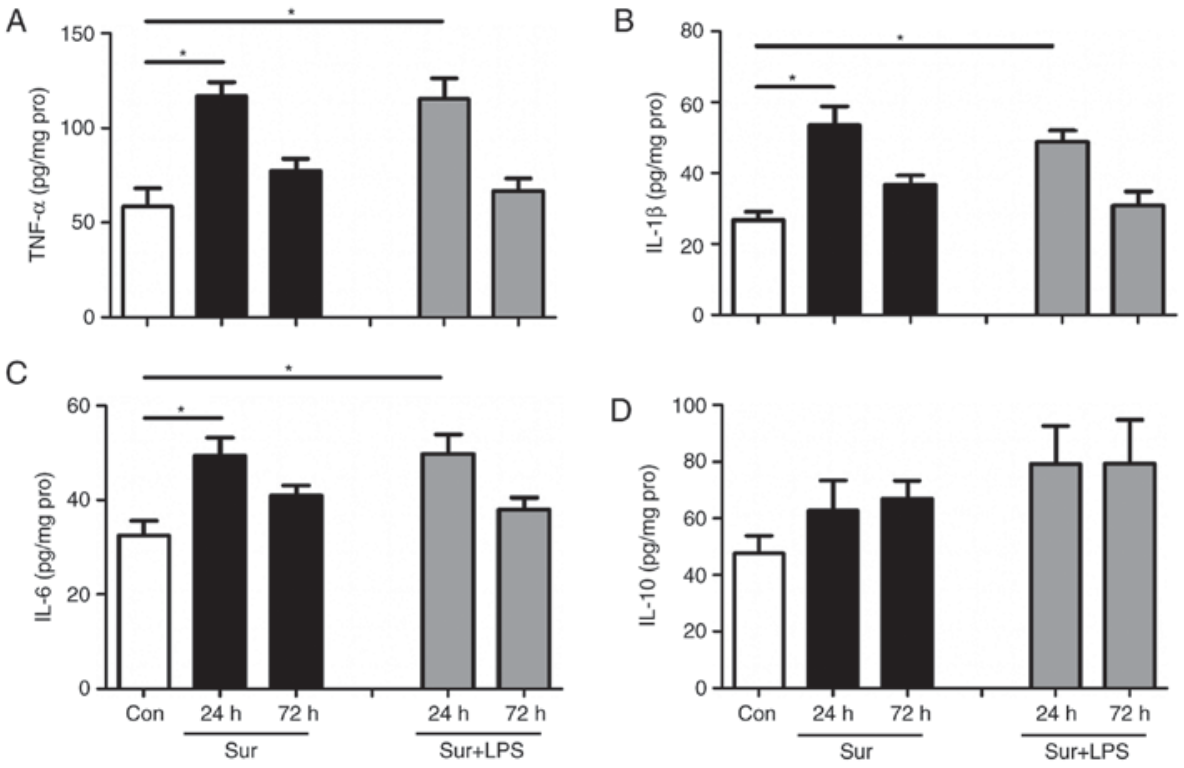

Figure 5. Effects of LPS administration at $6 \mathrm{~h}$ prior to surgery on the hippocampal levels of cytokines. A single low dose of LPS at $6 \mathrm{~h}$ prior to surgery did not alter the levels of (A) TNF- $\alpha$, (B) IL-1 $\beta$, (C) IL-6 or (D) IL-10. "P<0.05. Con, control; Sur, surgery; LPS, lipopolysaccharide, IL, interleukin; TNF, tumor necrosis factor.

low-dose LPS administration did not further increase the expression levels of the assessed cytokines in the hippocampus, measured at 24 and $72 \mathrm{~h}$ post-surgery, when compared with the surgery group $\left(\mathrm{TNF}-\alpha\right.$ : $\mathrm{F}_{(4,25)}=7.596, \mathrm{P}<0.001$; IL-1 $\beta$ : $\mathrm{F}_{(4.25)}=11.135, \mathrm{P}<0.001 ;$ IL-6: $\mathrm{F}_{(4.25)}=7.028, \mathrm{P}=0.001 ; \mathrm{IL}-10$ : $F_{(4,25)}=0.896, P=0.481$; Fig. 6).

Low-dose LPS preconditioning reverses surgery-induced microglial activation, depending on the regime of LPS administration. Since microglial cells are among the principal immune cells of the brain involved in the amplified neuroinflammatory response during the development of POCD, the present study evaluated whether low-dose LPS preconditioning was able to modulate the activity of microglia. Similar to the results of the analysis of cytokine expression, surgery induced a significant increase in the number of hippocampal IBA1-positive cells in the hippocampus, which was reversed by repeated LPS administration at $72 \mathrm{~h}$ or a single LPS administration at $24 \mathrm{~h}$ prior to surgery. Consistent with the above results, one single low-dose LPS administration at $6 \mathrm{~h}$ or immediately prior to surgery did not further increase the number of IBA1-positive cells in the hippocampus (Fig. 7). 
A

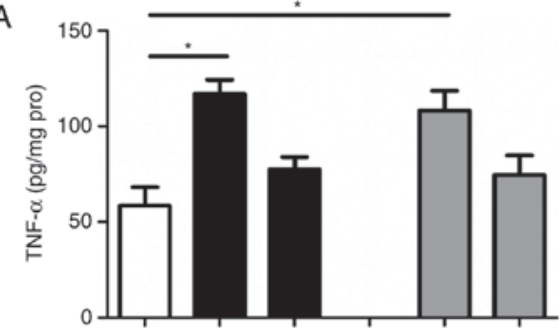

C

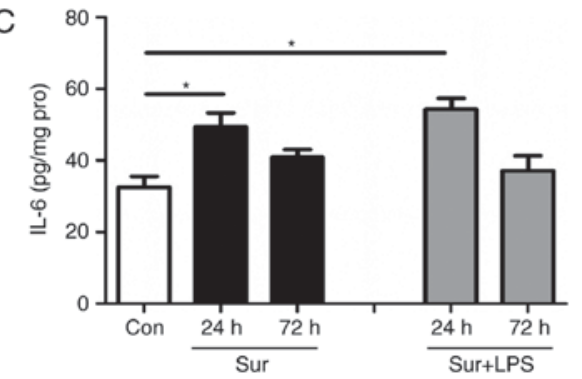

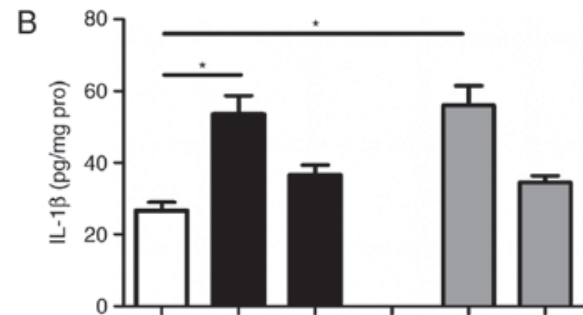

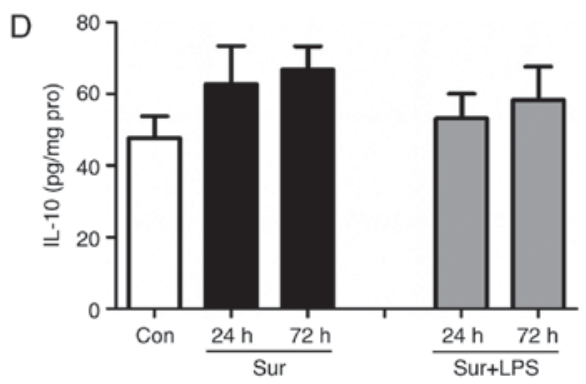

Figure 6. Effects of LPS administration at $0 \mathrm{~h}$ prior to surgery on the hippocampal levels of cytokines. Single low-dose LPS administration immediately prior to surgery did not further increase the levels of (A) TNF- $\alpha$, (B) IL-1 $\beta$, (C) IL-6 or (D) IL-10in the hippocampus. "P<0.05. Con, control; Sur, surgery; LPS, lipopolysaccharide; IL, interleukin; TNF, tumor necrosis factor.

A
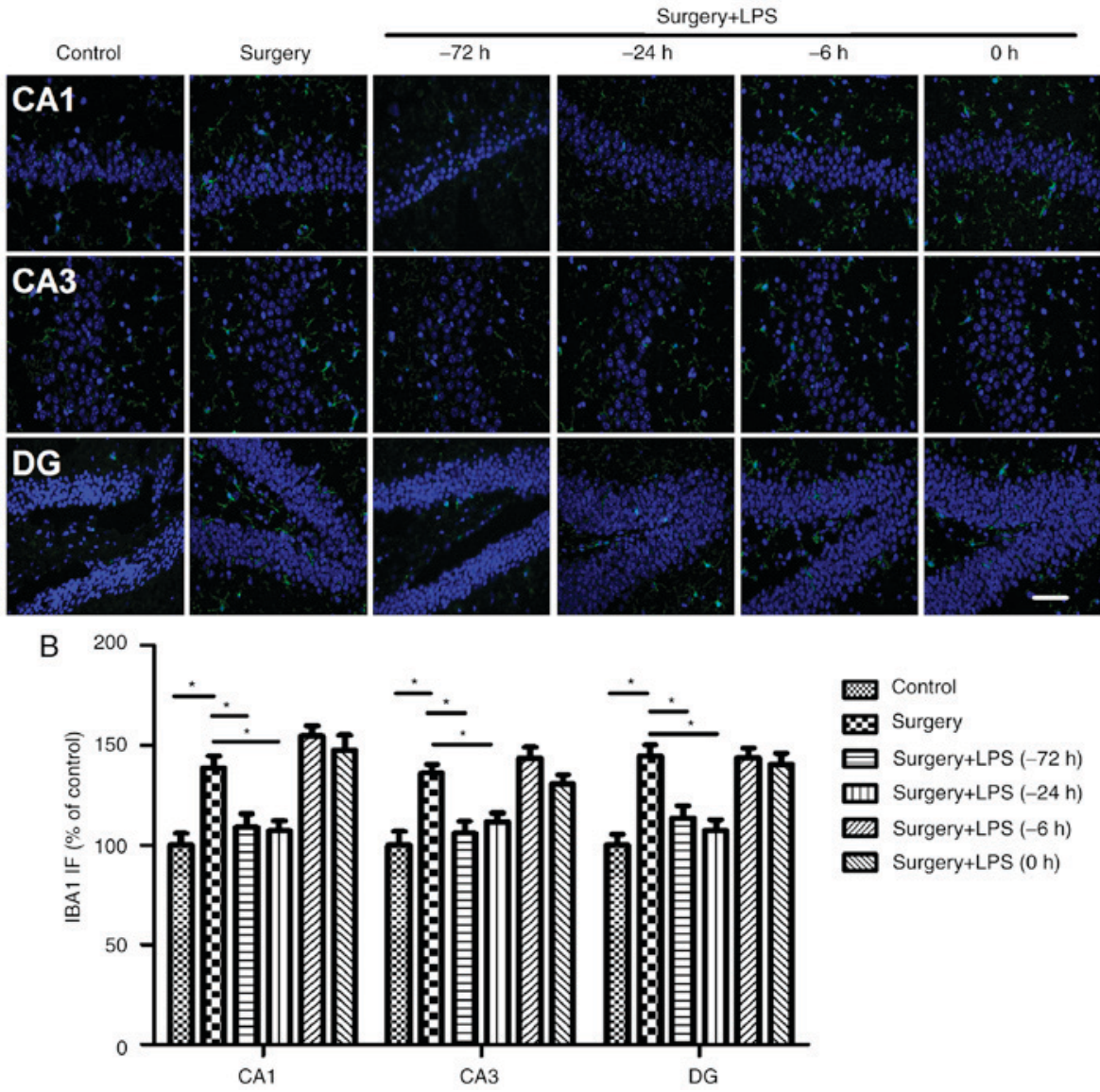

Figure 7. Low-dose LPS preconditioning reverses microglial activation, depending on the regime of LPS administration. (A) Representative images of IBA1 (green) and DAPI (blue) staining. (B) Quantification of IBA1 fluorescence in the hippocampus. Scale bar, $100 \mu \mathrm{m} .{ }^{*} \mathrm{P}<0.05$. LPS, lipopolysaccharide; IBA1, allograft inflammatory factor 1 ; DG, dentate gyrus.

\section{Discussion}

Substantial evidence has indicated that a dysregulated inflammatory response is causally linked to surgery-induced cognitive impairment (5-9). However, there remains no effective strategy to prevent or treat cognitive impairment resulting from surgery. In the present study, further evidence was produced to suggest that low-dose LPS preconditioning may 
protect against surgery-induced cognitive impairment in aging mice, as reflected by an improvement in hippocampal-dependent memory impairment and reduced production of pro-inflammatory mediators in the hippocampus.

Acute inflammation, as induced by aseptic trauma, including surgery, is important for optimal antimicrobial defense and healing, although an excessive inflammatory response may lead to exaggerated tissue damage (10). Peripheral surgery triggers an inflammatory response that may affect the central nervous system, contributing to neuroinflammation and POCD (11). Using a mouse model of surgery-induced cognitive decline $(16,17)$, which frequently leads to cognitive dysfunctions in humans, the results of the present study demonstrated that surgery induced significant cognitive impairment, concomitant with increased hippocampal levels of inflammatory cytokines. These data confirm previous findings demonstrating the role of neuroinflammation in the development of POCD (11). For example, inhibition of IL-1 receptors, or prophylactic treatment of mice with either a monoclonal antibody to TNF- $\alpha$ or by disabling HMGB1 with an inhibitory monoclonal antibody, may improve post-surgical cognitive decline (10-12,18). Although specific neutralization of the pro-inflammatory mediators appears promising in animal studies, anti-cytokine drugs are not selective to particular tissues and frequently produce undesirable side effects in clinical practice. In septic patients, the failure of anti-TNF- $\alpha$ - or IL-1 $\beta$-based therapies to decrease mortality has generated doubts as to whether cytokine-based treatments maybe effective (19). Moreover, cytokines have dual roles in sustaining the innate immune response and key physiological processes, including synaptic plasticity and tissue regeneration/healing (20). Therefore, the requirement for novel, selective treatment options for inflammation is urgent.

LPS is a surface component of the Gram-negative bacteria Toll-like receptor-4 ligand that activates the immune response to infections or other insults, leading to the induction of pro-inflammatory gene expression via the activation of a number of transcriptional pathways, including nuclear factor- $\kappa \mathrm{B}$ (21). Notably, animals that survive a sub-lethal exposure to the endotoxin are resistant to subsequent challenges with otherwise lethal doses of LPS during the first few days following the initial exposure, a phenomenon termed endotoxin tolerance (21). Increasing evidence indicates that endotoxin tolerance is a mechanism through which to limit the inflammatory response to subsequent stimuli to prevent excessive tissue damage (22). Although it has been suggested that long-term endotoxin tolerance may be a potentially detrimental condition, as it may hamper the ability to elicit a required immune response and thus lead to susceptibility to infection, it has been reported that immune function during LPS tolerance actually improves, as reflected by enhanced bacterial clearance and decreased mortality in LPS-tolerant animals challenged with live Gram-negative bacteria $(21,23)$. In addition, endotoxin tolerance confers resistance to inflammation and injury induced by a variety of other challenges, including myocardial infarction (24), kidney ischemia/reperfusion (25), neural ischemia (26) and traumatic spinal cord injury (14).

In the present study, it was demonstrated that repeated $(72 \mathrm{~h}$ prior to surgery) and single low-dose LPS preconditioning ( $24 \mathrm{~h}$ prior to surgery) abolished the signs of neuroinflammation. However, only single low-dose LPS preconditioning at $24 \mathrm{~h}$ prior to surgery improved the subsequent cognitive impairment following surgery. A possible explanation for these data maybe that repeated LPS administration itself may induce cognitive decline, which may offset the beneficial effects produced by endotoxin tolerance. Although the surgery-induced increased expression of hippocampal TNF- $\alpha$, IL-1 $\beta$ and IL- 6 did not coincide with a time-point when animals exhibited significant hippocampus-dependent cognitive impairment, it is possible that the anti-inflammatory effects of endotoxin tolerance produced by the LPS preconditioning in the early phase prevented the occurrence of subsequent events leading to delayed cognitive dysfunction. In addition, it was observed that LPS administration at $6 \mathrm{~h}$ or immediately prior to surgery did not exert marked beneficial effects. It has been suggested that the development of tolerance requires de novo protein synthesis (27), providing a possible explanation for the requirement of a time lag for tolerance to develop. In addition, it has been reported that LPS may induce sensitization and tolerance to subsequent oxygen-glucose deprivation (OGD), depending on the time interval between the insults, in organotypic hippocampal slice cultures: At a $0 \mathrm{~h}$ time interval between insults, LPS sensitized all neuronal regions of the hippocampus to subsequent OGD, whereas a time interval of $72 \mathrm{~h}$ resulted in ischemic tolerance (28). These observations confirm previous findings that the biological effects of LPS preconditioning depend on the time of preconditioning, the type of tissue challenged and the dose of LPS administered.

Although the mechanisms underlying LPS-induced tolerance remain unclear, accumulating evidence has implicated a role for pro-inflammatory cytokines, mediated by the activation of counter-regulatory mechanisms that result in an immune-suppressed state, characterized by lower levels of released cytokines upon a second challenge (21-24). In the present study, LPS preconditioning prevented the elevation of IL-1 $\beta$, TNF- $\alpha$ and IL- 6 expression in the hippocampus of mice at 1 day post-surgery. In addition, LPS preconditioning facilitates an inflammatory response to surgical trauma, albeit a less pronounced response, indicating that it may be a means to limit excessive inflammatory responses and tissue damage. In general, repeated and increased LPS dose injections may provide a greater suppression of the pro-inflammatory response (29). Notably, the present study suggested that one single dose of LPS administration at $24 \mathrm{~h}$ prior to surgery may be sufficient to induce a certain level of tolerance to subsequent surgical trauma, as evidenced by markedly reduced hippocampal pro-inflammatory cytokine expression. In addition, increased reactivity to an immune insult has been well characterized in aged subjects $(30,31)$, providing one explanation for the capacity of a lower dose of LPS to induce endotoxin tolerance. Other mechanisms, including reduced gliosis and antioxidant properties, have been reported to be involved in the mechanisms underlying endotoxin tolerance $(13,15)$. It was previously demonstrated that LPS preconditioning selectively inhibited the M1 response in an animal model of traumatic brain injury (13). Since LPS preconditioning regulates microglial phenotypic alterations, it provides a novel direction for future investigation of the mechanisms underlying the protective effects of endotoxin tolerance. Despite great clinical interest in LPS tolerance, the molecular events that underlie LPS tolerance remain to be completely understood, although Toll-like 
receptor desensitization and the suppression of the inflammatory signaling pathways via epigenetic reprogramming have been implicated (29). In order to have a comprehensive view of this phenomenon, an improved understanding of the mechanisms that underlie LPS tolerance ought to be determined.

In conclusion, the results of the present study suggested that LPS preconditioning may result in a reduced, although not inhibited, inflammatory response to subsequent surgical trauma. Since the majority of operations are planned, modulation of the dysregulated inflammatory response with LPS or other immune stimulators may afford protection against surgery-induced brain injury in patients who are at a higher risk of POCD and other neurodegenerative conditions.

\section{Acknowledgements}

The present study was financially supported by grants from the National Basic Research Program of China (grant no. 2013CB835100) and the National Natural Science Foundation of China (grant no. 81222013).

\section{References}

1. Steinmetz J, Christensen KB,Lund T, Lohse N and Rasmussen LS; ISPOCD Group: Long-term consequences of postoperative cognitive dysfunction. Anesthesiology 110: 548-555, 2009.

2. Monk TG, Weldon BC, Garvan CW, Dede DE, van der Aa MT, Heilman KM and Gravenstein JS: Predictors of cognitive dysfunction after major noncardiac surgery. Anesthesiology 108: 18-30, 2008.

3. Moller JT, Cluitmans P, Rasmussen LS, Houx P, Rasmussen H, Canet J, Rabbitt P, Jolles J, Larsen K, Hanning CD, et al: Long-term postoperative cognitive dysfunction in the elderly ISPOCD1 study. ISPOCD investigators. International study of post-operative cognitive dysfunction. Lancet 351: 857-861, 1998

4. Krenk L, Rasmussen LS and Kehlet H: New insights into the pathophysiology of postoperative cognitive dysfunction. Acta Anaesthesiol Scand 54: 951-956, 2010.

5. Qiu LL, Ji MH, Zhang H, Yang JJ, Sun XR, Tang H, Wang J, Liu WX and Yang JJ: NADPH oxidase 2-derived reactive oxygen species in the hippocampus might contribute to microglial activation in postoperative cognitive dysfunction in aged mice. Brain Behav Immun 51: 109-118, 2016.

6. Tan H, Cao J, Zhang J and Zuo Z: Critical role of inflammatory cytokines in impairing biochemical processes for learning and memory after surgery in rats. J Neuroinflammation 11: 93, 2014.

7. Hovens IB, Schoemaker RG, van der Zee EA, Absalom AR, Heineman E and van Leeuwen BL: Postoperative cognitive dysfunction: Involvement of neuroinflammation and neuronal functioning. Brain Behav Immun 38: 202-210, 2014.

8. Li Z, Liu F, Ma H, White PF, Yumul R, Jiang Y, Wang N and Cao X: Age exacerbates surgery-induced cognitive impairment and neuroinflammation in Sprague-Dawley rats: The role of IL-4. Brain Res 1665: 65-73, 2017.

9. Li N, Zhang X, Dong H, Hu Y and Qian Y: Bidirectional relationship of mast cells-neurovascular unit communication in neuroinflammation and its involvement in POCD. Behav Brain Res 322: 60-69, 2017.

10. Terrando N, Monaco C, Ma D, Foxwell BM, Feldmann M and Maze M: Tumor necrosis factor-alpha triggers a cytokine cascade yielding postoperative cognitive decline. Proc Natl Acad Sci USA 107: 20518-20522, 2010.

11. Vacas S, Degos V, Tracey KJ and Maze M: High-mobility group box 1 protein initiates postoperative cognitive decline by engaging bone marrow-derived macrophages. Anesthesiology 120: 1160-1167, 2014.

12. Terrando N, Yang T, Wang X, Fang J, Cao M, Andersson U, Erlandsson HH, Ouyang W and Tong J: Systemic HMGB1 neutralization prevents postoperative neurocognitive dysfunction in aged rats. Front Immunol 7: 441, 2016.
13. Turner RC, Naser ZJ, Lucke-Wold BP, Logsdon AF, Vangilder RL, Matsumoto RR, Huber JD and Rosen CL: Single low-dose lipopolysaccharide preconditioning: Neuroprotective against axonal injury and modulates glial cells. Neuroimmunol Neuroinflamm 4: 6-15, 2017.

14. Stevens SL, Leung PY, Vartanian KB, Gopalan B, Yang T, Simon RP and Stenzel-Poore MP: Multiple preconditioning paradigms converge on interferon regulatory factor-dependent signaling to promote tolerance to ischemic brain injury. J Neurosci 31: 8456-8463, 2011.

15. Li W, Jiang D, Li Q, Yao S, Sun X, Yang Y, Meng Z and Liu W: Lipopolysaccharide-induced preconditioning protects against traumatic spinal cord injury by upregulating Nrf2 expression in rats. Life Sci 162: 14-20, 2016.

16. Schaafsma W, Zhang X, van Zomeren KC, Jacobs S, Georgieva PB, Wolf SA, Kettenmann H, Janova H, Saiepour N, Hanisch UK, et al: Long-lasting pro-inflammatory suppression of microglia by LPS-preconditioning is mediated by RelB-dependent epigenetic silencing. Brain Behav Immun 48: 205-221, 2015.

17. Zhang Z, Yuan H, Zhao H, Qi B, Li F and An L: PPAR $\gamma$ activation ameliorates postoperative cognitive decline probably through suppressing hippocampal neuroinflammation in aged mice. Int Immunopharmacol 43: 53-61, 2017.

18. Zhang Z, Li X, Li F and An L: Berberine alleviates postoperative cognitive dysfunction by suppressing neuroinflammation in aged mice. Int Immunopharmacol 38: 426-433, 2016.

19. Barrientos RM, Hein AM, Frank MG, Watkins LR and Maier SF: Intracisternal interleukin-1 receptor antagonist prevents postoperative cognitive decline and neuroinflammatory response in aged rats. J Neurosci 32: 14641-14648, 2012.

20. Dinarello CA: Anti-cytokine therapeutics and infections. Vaccine 21 (Suppl 2): S24-S34, 2003.

21. Terrando N, Gómez-Galán M, Yang T, Carlström M, Gustavsson D, Harding RE, Lindskog M and Eriksson LI: Aspirin-triggered resolvin D1 prevents surgery-induced cognitive decline. FASEB J 27: 3564-3571, 2013.

22. Murphey ED, Fang G and Sherwood ER: Endotoxin pretreatment improves bacterial clearance and decreases mortality in mice challenged with Staphylococcus aureus. Shock 29: 512-518, 2008.

23. Arbibe L and Sansonetti PJ: Epigenetic regulation of host response to LPS: Causing tolerance while avoiding Toll errancy. Cell Host Microbe 1: 244-246, 2007.

24. Fensterheim BA, Guo Y, Sherwood ER and Bohannon JK: The cytokine response to lipopolysaccharide does not predict the host response to infection. J Immunol 198: 3264-3273, 2017.

25. Fernández-Ruiz I, Arnalich F, Cubillos-Zapata C, Hernández-Jiménez E, Moreno-González R, Toledano V, Fernández-Velasco M, Vallejo-Cremades MT, EstebanBurgos L, de Diego RP, et al: Mitochondrial DAMPs induce endotoxin tolerance in human monocytes: An observation in patients with myocardial infarction. PLoS One 9: e95073, 2014.

26. Dai Y, Jia P, Fang Y, Liu H, Jiao X, He JC and Ding X: miR-146a is essential for lipopolysaccharide (LPS)-induced cross-tolerance against kidney ischemia/reperfusion injury in mice. Sci Rep 6: 27091, 2016.

27. Vartanian KB, Stevens SL, Marsh BJ, Williams-Karnesky R, Lessov NS and Stenzel-Poore MP: LPS preconditioning redirects TLR signaling following stroke: TRIF-IRF3 plays a seminal role in mediating tolerance to ischemic injury. J Neuroinflammation 8: 140, 2011.

28. Eklind S, Mallard C, Arvidsson $\mathrm{P}$ and Hagberg $\mathrm{H}$ : Lipopolysaccharide induces both a primary and a secondary phase of sensitization in the developing rat brain. Pediatr Res 58: 112-116, 2005.

29. Markus T, Cronberg T, Cilio C, Pronk C, Wieloch T and Ley D: Tumor necrosis factor receptor-1 is essential for LPS-induced sensitization and tolerance to oxygen-glucose deprivation in murine neonatal organotypic hippocampal slices. J Cereb Blood Flow Metab 29: 73-86, 2009.

30. Seeley JJ and Ghosh S: Molecular mechanisms of innate memory and tolerance to LPS. J LeukocBiol 101: 107-119, 2017.

31. Kohman RA, Crowell B and Kusnecov AW: Differential sensitivity to endotoxin exposure in young and middle-age mice. Brain Behav Immun 24: 486-492, 2010. 